\title{
Survey of the distribution of lesion size in multiple sclerosis: implication for the measurement of total lesion load
}

\author{
Liqun Wang, H-M Lai, A J Thompson, D H Miller
}

\begin{abstract}
Objectives-Quantitative measurement of lesion load on proton density or T2 weighted brain MRI in multiple sclerosis is a widely used marker of disease progression in treatment trials and natural history studies. However, it has proved difficult to obtain highly reproducible measurements. Several factors account for this, one of which is uncertainties in lesion identification, particularly very small white matter abnormalities. This paper aims to ascertain the significance of very small white matter abnormalities in the measurement of lesion load in multiple sclerosis.

Methods-All visible lesion areas identified by an experienced observer on proton density weighted spin echo brain MRI with $5 \mathrm{~mm}$ thick slices were measured by using a contouring technique in 15 patients with secondary progressive multiple sclerosis (SPMS) and 13 with relapsing remitting multiple sclerosis (RRMS). The size distribution of these lesions was analysed.

Results $-80 \%$ of the number of the lesions were smaller than $80 \mathrm{~mm}^{2}$. Lesions that were smaller than $10 \mathrm{~mm}^{2}$ (equivalent diameter $<3.5 \mathrm{~mm}$ ) made up nearly $20 \%$ of all lesions; their relative contribution to the total lesion load varied from $0.0-5.7 \%$ (mean $=1.1 \%$, median $=0.65 \%)$ in individual patients, and was larger when the total lesion load was smaller $(r=-0.65$, $\mathbf{P}<0.001)$. Median lesion size was significantly smaller in the SPMS group than the RRMS group.

Conclusions-The results suggest that it is prudent to identify and measure small lesions in evaluating treatment effects, and that measures are undertaken (for
\end{abstract}

Institute of Neurology, Queen Square, London, UK

L Wang

H-M Lai

A J Thompson

D H Miller

Correspondence to: Professor D H Miller, NMR Research Unit, Institute of Neurology, Queen Square, London WC1N 3BQ, UK.

Received 7 August 1996 and in revised form 27 March 1997 Accepted 4 April 1997

Quantitative measurement of lesion load on T2 weighted brain MRI in multiple sclerosis is a widely used marker of disease progression in treatment trials. ${ }^{12}$ However, it has been difficult to measure lesion load with a high degree of reproducibility. The coefficient of variation of measurement of lesion load reported in the literature ranges from $2 \%-34 \% .^{3-7}$ This high variability has multiple causes including differences in measurement techniques, image quality, slice thickness, scanner and rater performances, and lesion identification strategy.

At present, no gold standard exists for lesion identification and delineation. The consensus review of experienced neuroradiologists has been suggested as a best available "silver" standard, ${ }^{8}$ but inconsistency exists, even in the decisions of one radiologist made on different occasions. One particular difficulty is the lack of consensus on whether very small white matter abnormalities on MRI are significant and should be included as lesions for lesion volume measurement. Throughout this report we use the term small lesion to refer to white matter lesions in multiple sclerosis, seen on MRI, that are no larger than $3.5 \mathrm{~mm}$ in diameter. We have surveyed a data base of multiple sclerosis lesions to describe the distribution of lesion sizes to elucidate whether small lesions should be included and to what extent they influence overall lesion load measurement.

\section{Materials}

PATIENTS

Twenty eight patients with clinically definite multiple sclerosis ${ }^{9}$ were studied. The patients had all been recruited into a double blind, placebo controlled trial of anti-CD4 antibody; full details of the criteria for entry to this study are published elsewhere. ${ }^{10}$ Fifteen of them had secondary progressive multiple sclerosis (SPMS) and 13 relapsing remitting multiple sclerosis (RRMS). Table 1 shows their clinical data.

MRI

Conventional dual spin echo axial proton density and T2 weighted MRI images were acquired for all patients on a GE Signa 1.5T scanner, using a birdcage head coil. The imaging parameters were: SE 2000/34/90, $5 \mathrm{~mm}$ thick contiguous interleaved slices, $256 \times 256$ matrix, $24 \mathrm{~cm}$ field of view, 1 excitation. The total number of slices was 28 for each echo. The scans were corrected for non-uniformity of the coil before being analysed. ${ }^{11}$
ANALYSIS

The lesions were identified and then segmented on the proton density weighted scans-under the supervision of a radiologist (MG-C) - by a neurologist who had two years 
Table 1 Patient information

\begin{tabular}{lllll}
\hline Subgroup & Sex (F:M) & Median EDSS & Mean disease duration $(y)$ & Mean age $(y)$ \\
\hline RRMS & $8: 5$ & $3.5(2.0-6.0)$ & $6.9(1-22)$ & $35.5(26-47)$ \\
SPMS & $7: 8$ & $6.0(3.5-8.0)$ & $10.3(2-29)$ & $40.5(30-53)$ \\
\hline
\end{tabular}

RRMS=Relapsing-remitting multiple sclerosis; SPMS=secondary progressive multiple sclerosis; EDSS=expanded disability status scale..$^{15}$

Values in parentheses are range.

of experience in lesion load measurement (H-ML). The technique used for lesion segmentation is a contouring method provided by D Plummer (Dispimage@DPlummer, University College London). This semiautomated, local thresholding method has been fully described elsewhere, ${ }^{4}$ and was shown to provide a good within and between rater reproducibility. Lesion areas were obtained by calculating the area of the regions of interests identified. The rater used both the proton density weighted and T2 weighted images to identify multiple sclerosis lesions but segmentation was performed only on proton density images. The rater was able to view the neighbouring slices simultaneously on the computer workstation to assist lesion identification when measuring but was blinded to the patients' clinical subgroup. (Small hyperintense areas of uncertain cause-for example, possible small blood vessels - were not included in the analysis.)

The within rater coefficient of variation of measurement of lesion volume was $3.0 \%$. This was obtained from 10 measurements on five patients, two measurements on each patient with a two week interval in between.

For this study, we have defined lesions as regions or areas of signal abnormality seen on single slices. These regions or lesion areas are the nearest approximation we have to actual pathological lesions on two dimensional MRI. If a single large pathological lesion actually extended over several slices we have counted it as a separate MRI lesion on every slice that it appeared.

The distribution in size for 1568 lesions in 15 patients with SPMS and 1198 lesions in 13 patients with RRMS was investigated. Lesion area was measured after it had been delineated using the contouring technique on a slice by slice basis. The nominal diameter of a lesion was then calculated by assuming that it had a circular shape. The purpose of calculating a nominal diameter was to help understand the comparisons of lesion sizes. The nominal diameter was not used in calculating the lesion area. Figure 1 shows an example of lesions delineated using the contouring technique. Lesion volumes were calculated by multiplying the slice thickness by the lesion areas of delineated lesion regions only because it is the

Table 2 Results of analysis

\begin{tabular}{llll}
\hline Measurement & RRMS & SPMS & Difference $^{*}$ \\
\hline Total lesion volume $(\mathrm{ml})$ & $35.7(2.1-116.0)$ & $42.4(4.2-151.0)$ & NS \\
Median lesion size $\left(\mathrm{mm}^{2}\right)$ & $40.9(9.7-1011.6)$ & $32.5(1.8-3549.9)$ & $\mathrm{P}<0.0001$ \\
Median No of lesions & $75(15-227)$ & $96(25-242)$ & NS \\
†SLV/TLV (\%) & $1.0(0.0-4.7)$ & $1.3(0.0-5.7)$ & NS \\
\hline
\end{tabular}

$\star$ Significance inferred from Mann-Witney rank sum test.

+ SLV/TLV $=$ small lesion volume $\times 100 /$ total lesion volume.

Results are given as mean (range).

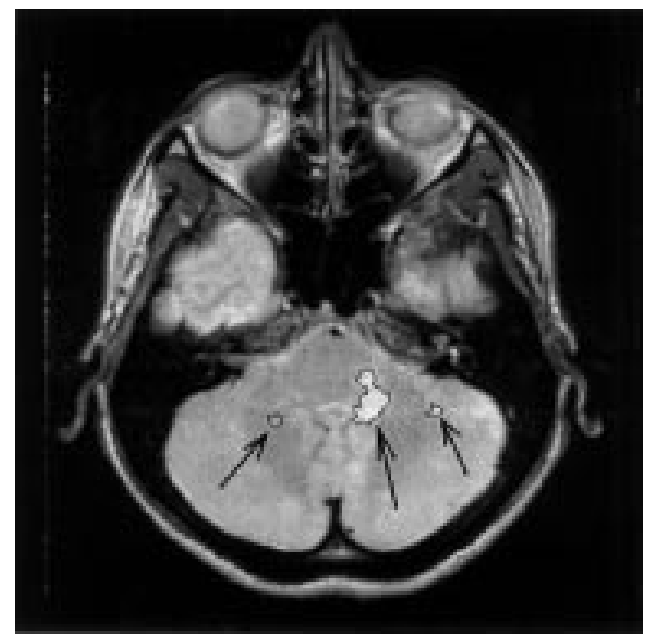

Figure 1 Example of lesions delineated using the contouring technique. The lesion sizes are 16.2, 129.7, and $17.2 \mathrm{~mm}^{2}$ respectively from left, middle, and right. The corresponding nominal diameters are $4.5,12.9$, and 4.7
$\mathrm{~mm}$.

conventional unit of measurment. All comparisons in size were carried out on lesion areas.

\section{Results}

Figure 2 depicts the distribution of the percentage of lesions of different sizes. It is evident that the distribution is skewed towards smaller size.

About $80 \%$ of all the lesions were smaller than $8 \mathrm{~mm}$ in nominal diameter. The average lesion size was about $36 \mathrm{~mm}^{2}(7 \mathrm{~mm}$ in nominal diameter). About $20 \%$ of lesions were small (nominal diameter $<3.5 \mathrm{~mm}$ ). Their contribution to the total lesion loads varied considerably from one subject to another (range $0-5.7 \%$; mean $1.1 \%$; fig 3 ); it was largest when total lesion loads were small. Thus the percentage contribution of small lesions to the total lesion volume correlated inversely with the total lesion volume (Spearman coefficient of correlation $r=-0.65, \mathrm{P}<0.001)$.

Table 2 shows the results for the two clinical subgroups. Lesion sizes were on average smaller in the SPMS group ( $<<0.001$, MannWhitney rank sum test) although there were wide overlapping ranges of individual lesion sizes. The total lesion volumes in each subgroup were not significantly different.

\section{Discussion and conclusions}

Our evaluation of lesion size was based on the areas of the regions delineated on a slice by slice basis. This practice tends to bias towards the smaller lesion areas, as a confluent biological lesion going through several slices is treated as several smaller lesion regions. This may partly explain why the distribution of the number of lesions was skewed towards smaller sizes. However, as most of the lesion quantification methods used currently are based on region identification on individual slices on two dimensional images, we think that this approach is relevant.

Our findings suggest that small lesions should not be ignored in measurement of lesion volume. They can be quantitatively 


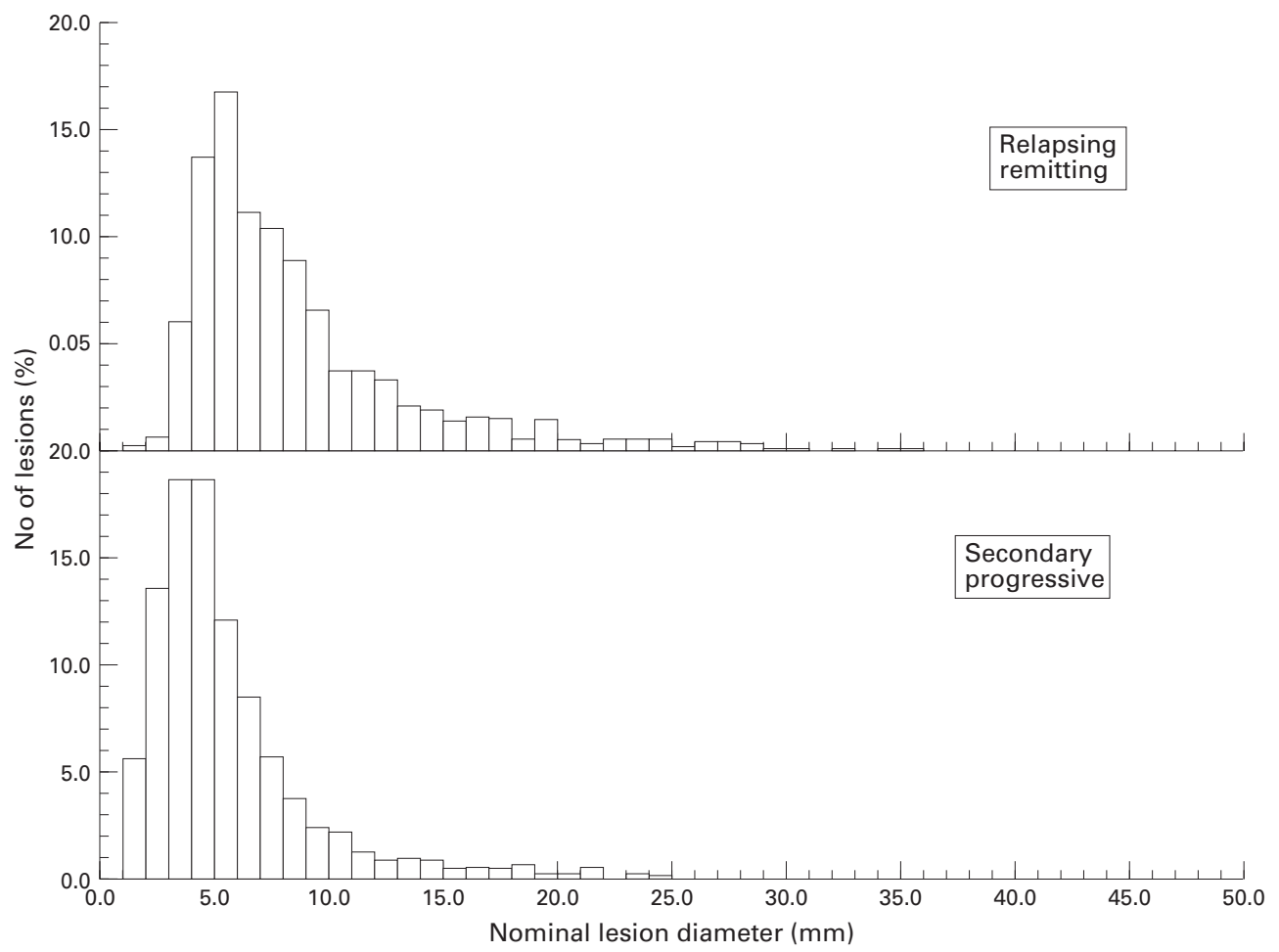

Figure 2 Distribution of the number of lesions in size.

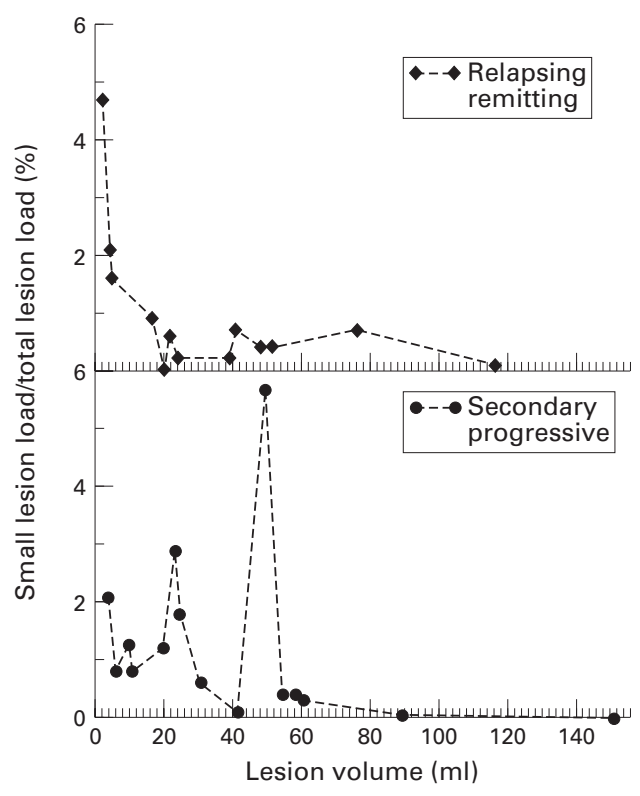

Figure 3 Contribution of the load of small lesions $(<10$ $\mathrm{mm}^{2}$ ) to the total lesion load.

important, especially in patients with small total lesion loads. A subgroup of patients with multiple sclerosis in whom small lesions are especially prominent are those with primary progressive disease; ${ }^{12}{ }^{13}$ it follows that quantification of small lesions in this subgroup should always be attempted. There may also be a case for considering patients with small, moderate, and large lesion loads separately.

The current study applies to $5 \mathrm{~mm}$ thick slices. Recent work on $3 \mathrm{~mm}$ thick slices $^{14}$ found an $8 \%$ increase in total lesion load which is likely to reflect a contribution from small lesions. The increasing use of thinner slices for clinical trials is likely to result in identification of additional small lesions with important effects on measured lesion load; in the near future sequences such as three dimensional fast spin echo should provide isotropic resolution.

In the SPMS group a slightly higher proportion of the total lesion load was made up by small lesions and the median lesion size was significantly smaller compared with the RRMS group (table 2). Patients with SPMS also exhibited a different pattern of small lesion load contribution in relation to total lesion load from that of RRMS, as shown graphically in fig 3. Small lesions should be investigated more fully to ascertain whether their number and proportion are related to the clinical evolution of the disease. It is not inconceivable, for example, that the accumulation of mainly small lesions, none of which alone is sufficient to cause an acute relapse, might result in a slowly progressive increase in clinical disability. Future prospective studies are needed to consider this question.

The Institute of Neurology NMR Research Unit is funded by the Multiple Sclerosis Society of Great Britain and Northern Ireland. We thank Dr Mary Gawn-Cain for her radiological input.

1 Paty DW, Li DKB, the INFB MS Study Group. Interferon $\beta-1 \mathrm{~b}$ is effective in relapsing remitting multiple sclerosis. ii. MRI analysis results of a multicenter, randomized, doubleblind, placebo controlled trial. Neurology 1993;43:662-7.

2 Miller DH, Albert PS, Barkhof F, et al. Guidelines for the use of magnetic resonance techniques in monitoring the treatment of multiple sclerosis. Ann Neurol 1996;39:6-16.

3 Clarke LP, Velthuizen RP, Camacho MA, et al. MRI segmentation: methods and applications. Magn Reson Imaging 1995;13:343-68.

4 Grimaud JP, Lai M, Plummer D, et al. Quantification of MRI lesion load in multiple sclerosis: a comparison of three computer-assisted techniques. Magn Reson Imaging 1996; 14:495-505.

5 Goodkin DE, Ross JS, et al. Magnetic resonance imaging lesion enlargement in multiple sclerosis. Arch Neurol 1992; 49:261-3. 
6 Fillipi M, Horsfield MA, Tofts PS, et al. Quantitative assessment of MRI lesion load in monitoring the evolution of multiple sclerosis. Brain 1995;118:1601-12.

7 Wang, L, Thorpe JW, Tofts PS. Evaluation of cluster analysis in multiple sclerosis lesion segmentation. Proceedings of the Annual Conference of Society of Magnetic Resonance in Medicine 1995;2:709.

8 Evans A, Frank JA, Antel J, Miller DH. The role of MRI in clinical trials of multiple sclerosis: comparison of image processing techniques. Ann Neurol 1997;41:125-32.

9 Poser CM, Paty DW, Scheinberg L, et al. New diagnostic criteria for multiple sclerosis: guidelines for research protocols. Ann Neurol 1983;13:227-31.

10 van Oosten BW, Lai M, Barkhof F, et al. A phase II trial of anti-CD4 antibodies in the treatment of multiple sclerosis . Multiple Sclerosis 1996;1:339-42.
11 Wicks DAG, Barker GJ, Tofts PS. Correction of intensity non-uniformity in MR images of any orientation. Magn Reson Imaging 1992;11:183-96.

12 Thompson AJ, Kermode AG, MacManus DG, et al. Patterns of disease activity in multiple sclerosis: clinical and magnetic resonance imaging study. BMF 1990;30:631-4.
Thompson AJ, Kermode AG, Wicks DAG, et al. Major differences in the dynamics of primary and secondary progressive MS. Ann Neurol 1991;29:53-62.

4 Filippi M, Horsfield MA, Campi A, et al. Resolutiondependent estimates of lesion volumes in magnetic resonance imaging studies of the brain in multiple sclerosis. Ann Neurol 1995;38:749-54.

15 Kurtzke JF. Rating neurological impairment in multiple sclerosis: an expanded disability status scale (EDSS). Neurology 1983;33:1444-52. 